\title{
MULTICRITERIA COMBINATORIAL OPTIMIZATION PROBLEMS ON A SET OF POLYPERMUTATIONS
}

\author{
L. N. Kolechkina ${ }^{a}$ and E. A. Rodionova ${ }^{b}$
}

UDC 519.85

The studies on multicriteria combinatorial optimization are continued. A possible approach to solving multicriterion problems is developed and substantiated. An algorithm is developed and implemented. Some peculiarities of efficient solutions to multicriterion problems are described.

Keywords: multicriterion combinatorial problems, polypermutations, efficient alternative, Paretooptimal solutions.

\section{INTRODUCTION}

Studying the properties of discrete problems [1-8] and developing methods to solve them is of current importance. It is rather important to analyze the class of combinatorial problems separated out from discrete ones.

In solving practical problems, mathematical models of combinatorial optimization problems are frequently used [3, 4 , 8]. Depending on the complexity of an applied problem, not one but several optimization criteria that cannot be combined into one can be considered simultaneously. It is often necessary to take into account the combinatorial properties of the set of admissible values. Thus, it is expedient to search for the solution of multicriteria optimization problems in view of the combinatorial properties of the domain of admissible values. The above-mentioned problems are scantily studied and complicated; therefore, their study is of current importance.

The studies $[1,2,5,7]$ analyze modeling problems in economy and engineering and the use of models of discrete multicriteria optimization. The properties of combinatorial optimization problems with vector criteria, their complexity, solvability, and stability and algorithmic problems of their solution are investigated. However, theoretical studies and practical experience have shown high complexity of discrete, in particular, combinatorial optimization problems.

Nowadays, the properties of combinatorial sets are analyzed and methods of solving combinatorial optimization problems are developed. However, their possible application to a certain class of problems is usually studied. Searching for general approaches and solution techniques for solving multicriterion problems on a combinatorial set, in particular, on a set of polypermutations, is yet to be investigated and thus is topical.

The paper continues the studies described in [1-8]; it deals with a class of multicriteria optimization problems on a combinatorial set of polypermutations and develops an approach to solve them.

\section{PROBLEM STATEMENT}

Let us consider multicriterion problems with account for the combinatorial properties of the region of feasibility. In practice, such problems arise when it is necessary to formalize some conditions as criteria (these criteria cannot be united) with optimal values to be found.

Let us consider a multicriterion unconditional problem on polypermutations. Denote by $N_{m}$ and $N_{s}$ the sets of $m$ and $s$ first natural numbers, respectively: $N_{m}=\{1, \ldots, m\}$ and $N_{s}=\{1, \ldots, s\}$. The criteria to be optimized can be represented by a set of functions:

${ }^{\mathrm{a}}$ V. M. Glushkov Institute of Cybernetics, National Academy of Sciences of Ukraine, Kyiv, Ukraine, ludapl@ukr.net. ${ }^{\mathrm{b}}$ Poltava Institute of Business, Poltava, Ukraine, rodionovaoa@mail.ru. Translated from Kibernetika i Sistemnyi Analiz, No. 2, pp. 152-160, March-April 2008. Original article submitted April 5, 2007. 


$$
\begin{gathered}
f_{1}(x)=\sum_{j=1}^{k} c_{j}^{1} x_{j} \rightarrow \min , \\
f_{2}(x)=\sum_{j=1}^{k} c_{j}^{2} x_{j} \rightarrow \min , \\
\ldots \ldots \ldots \ldots \ldots \\
f_{s}(x)=\sum_{j=1}^{k} c_{j}^{s} x_{j} \rightarrow \min , \\
f_{s+1}(x)=\sum_{j=1}^{k} c_{j}^{s+1} x_{j} \rightarrow \max , \\
\ldots \ldots \ldots \ldots \ldots \\
f_{m}(x)=\sum_{j=1}^{k} c_{j}^{m} x_{j} \rightarrow \max .
\end{gathered}
$$

Therefore, $s$ functions out of $m$ should be minimized and $m-s$ functions should be maximized (however, in solving practical problems, a need often arise to decrease one criteria and increase other ones). There may be a special case where all the functions are maximized or minimized.

It is expedient to represent the set of functions (1) as a vector function

$$
F\left(-f_{1}, \ldots,-f_{s}, f_{s+1}, \ldots, f_{m}\right)
$$

whose maximum should be found.

The condition that solutions should belong to a combinatorial set can arise from the side conditions imposed on the variables in the problem formulation. In the mathematical model of problem (1), the condition is imposed that the solution should belong to a set of polypermutations of the form

$$
x=\left(x_{1}, \ldots, x_{k}\right) \in E_{\eta n}^{k s}(G, H) .
$$

As is known from [8], the set of polypermutations is equal to the set of nodes of the polyhedron of polypermutations, $E_{\eta n}^{k s}(G, H)=$ vert $\Pi_{\eta n}^{k s}(G, H)$, whose convex hull $\Pi_{\eta n}^{k s}(G, H)$ can be described by

$$
\left\{\begin{array}{l}
\sum_{j \in \omega^{i}} x_{j} \geq \sum^{\left|\omega^{i}\right|} g_{j}^{M_{i}}, \\
\sum_{j \in \omega^{i}} x_{j} \leq \sum^{\left|\omega^{i}\right|} g_{\eta_{i}-j+1}^{M_{i}} \forall \omega^{i} \subset M_{i} \quad \forall i \in N_{s} .
\end{array}\right.
$$

In view of all the above conditions, we can formulate the problem as follows: find the set of values (3) that is optimal for functions (2). Such a problem is called a multicriterion unconditional combinatorial problem on a set of polypermutations. If side conditions $D$ of the form

$$
A_{i j} x_{j} \leq b_{j}, \text { where } i \in N_{m}, j \in N_{k},
$$

are imposed on the set of admissible solutions, then problem (2), (3), (5) is a multicriterion combinatorial problem on polypermutations with additional constraints:

$$
X=D \cap \Pi_{\eta n}^{k s}(G, H)
$$




\section{APPROACH TO SOLVING COMBINATORIAL MULTICRITERION PROBLEMS}

The solution of multicriterion combinatorial problems is accompanied by finding an efficient solution from a comparison of alternatives on the set of objective functions. Such a solution may appear optimal for none of the objective functions; however, it is the best compromise in view of all the objective functions simultaneously.

Definition 1 [5]. A solution is efficient if the set of admissible solutions determined by the condition (4), (5) does not contain a solution $\bar{x}$ such that the inequalities

$$
\begin{aligned}
& f_{i}(\bar{x}) \geq f_{i}\left(x_{0}\right) \quad \forall i \in I_{1}, \\
& f_{i}(\bar{x}) \leq f_{i}\left(x_{0}\right) \quad \forall i \in I_{2}
\end{aligned}
$$

hold and at least one of them is strict.

This means that none of admissible solutions can improve the value of some objective function without worsening at least one of the remaining objective functions. An tfficient alternative is also called Pareto optimal [5]. All such alternatives constitute a set $P(F, X)$ of Pareto optimal solutions [6, 7]. According to [7], the statement

$$
x \in P(F, X) \Leftrightarrow\{y \in X \mid F(y) \geq F(x), F(y) \neq F(x)\}=\varnothing
$$

is true for any $x \in X$, where $F$ is the vector function (2), and $X$ is the set of admissible values of the function, which is determined by conditions (6) of the problem.

To determine the set of efficient solutions of the multicriteria combinatorial optimization problem, we will use the following theorem.

THEOREM 1 [8]. If $x^{*}=\left(x_{1}^{*}, \ldots, x_{l}^{*}\right)=\left(X_{1}^{*}, \ldots, X_{i}^{*}, \ldots, X_{s}^{*}\right)$, where $X_{j}^{*}=\left(x_{m_{j}+1}^{*}, \ldots, x_{m_{j}}^{*} k_{j}\right)$, then the following conditions are satisfied:

$$
\begin{gathered}
g_{1}^{M_{i}} \leq \ldots \leq g_{\eta_{i}}^{M_{i}}, \\
c_{m_{j}+1} \geq \ldots \geq c_{m_{j}+s_{j}} \geq 0>c_{m_{j}+s_{j}+1} \geq \ldots \geq c_{m_{j}+k_{j}}, \\
m_{0}=0, \quad k_{0}=0, \quad m_{j}=m_{j-1}+k_{j-1} \quad \forall j \in N_{s} .
\end{gathered}
$$

Then the minimum of the function $f(x)=\sum_{j=1}^{k} c_{j} x_{j}$ on the set of polypermutations $E_{\eta n}^{k s}(G, H)$ is attained at the point $x^{*}=\left(x_{1}, \ldots, x_{k}\right) \in E_{\eta n}^{k s}(G, H)$ that satisfies the conditions

$$
x_{m_{j}+1}^{*}=g_{i}^{M_{j}} \quad \forall i \in N_{s_{j}} ; \quad x_{m_{j}+s_{j}+1}^{*}=g_{\eta_{i}-r_{j}+i}^{M_{j}} \quad \forall i \in M_{r_{j}}, \forall j \in N_{s},
$$

where $r_{j}$ and $s_{j}$ satisfy the conditions

$$
r_{j}, s_{j} \in N_{k_{j}}^{0}, \quad r_{j}+s_{j}=k_{j} \quad \forall j \in N_{s} .
$$

The previous theorem yields the following optimality criterion for a multicriterion unconditional problem on a set of polypermutations.

THEOREM 2. Let $B_{i}$ be the set of permutations $\beta^{i}=\left(\beta_{1}^{i}, \ldots, \beta_{k}^{i}\right)$ that belong to the set $E_{k}\left(N_{k}\right)$ and satisfy the condition

$$
C_{\beta_{1}^{i}}^{i} \geq C_{\beta_{2}^{i}}^{i} \geq \ldots \geq C_{\beta_{s}^{i}}^{i} \geq 0>C_{\beta_{s+1}^{i}}^{i} \geq \ldots \geq C_{\beta_{k}^{i}}^{i}
$$

Then the set of solutions of the unconditional multicriterion problem is not empty if the set being the intersection of the sets $B_{i}$ found for criteria (1) is not empty:

$$
\bigcap_{i=1}^{m} B_{i}=B=\varnothing
$$

For any permutation $\beta=\left(\beta_{1}, \ldots, \beta_{k}\right) \in B$, a point $x^{*}=\left(x_{1}, \ldots, x_{k}\right) \in E_{\eta n}^{k s}(G, H)$ in the form (11) under conditions (8)-(10), (12) belongs to the set of solutions. 
Let us consider the following example that illustrates Theorem 2.

Given a multiset $G=\{1,2,3,3,3,4,5,6,7\}$ consisting of $\eta=9$ elements; therefore, $N_{9}=\{1,2,3,4,5,6,7,8,9\}$. Let $s=3$, we choose a partition of $N_{9}$ into sets $K_{1}=\{1,2,3\}, K_{2}=\{4,6\}, K_{3}=\{5,7,8,9\}$ and specify $k=4, k_{1}=1, k_{2}=1$, and $k_{3}=2$. Then the set of polypermutations can be represented as $E_{97}^{43}(G, H)$.

The following set of functions is given:

$$
\left\{\begin{array}{l}
f_{1}=5 x_{1}+x_{2}+10 x_{3}-x_{4} \rightarrow \min \\
f_{2}=2 x_{1}+x_{2}+3 x_{3}-2 x_{4} \rightarrow \min \\
f_{3}=3 x_{1}+2 x_{2}+4 x_{3}-x_{4} \rightarrow \min \\
f_{4}=7 x_{1}+5 x_{2}+7 x_{3}-3 x_{4} \rightarrow \min
\end{array}\right.
$$

Obviously, $C_{3}^{i} \geq C_{1}^{i} \geq \ldots \geq C_{2}^{i} \geq 0>C_{4}^{i} \forall i \in N_{4}$. The condition of Theorem 2 is satisfied; therefore, the point $x^{*}=(3,3,1,7) \in E_{97}^{43}(G, H)$ is optimal for each of the functions.

\section{COMBINED METHOD TO SOLVE MULTICRITERION COMBINATORIAL OPTIMIZATION PROBLEMS}

As we mentioned above, multicriterion combinatorial problems are of interest in solving some applied problems; however, the methods developed to date do not solve such problems adequately. A new approach to their solution is necessary. Let us consider a possible approach to solving such problems that combines two methods considered earlier: the method of constraints [2] and the method of combinatorial cut-off [3].

Let a set of objective functions written as a vector function (2) be given,

$$
f_{i}(x)=\sum_{j=1}^{k} c_{i j} x_{j}, \quad j \in N_{m},
$$

the first $s$ functions should be minimized, and the next $m-s$ maximized. Constraints (5) and the condition of belonging to the set of polypermutations (3) are imposed on the solution.

\section{Solution Algorithm}

1. Introduce an integer variable $q$ assuming $q=0$.

2. Write the condition of belonging to the set of polypermutations as the system of inequalities (4).

3. Combine system (4) with the system of additional linear constraints of problem (5).

4. Use Theorem 1 to determine solutions $x_{i}^{0}$ that belong to the set of polypermutations and optimize the $i$ th objective function $x_{i \max }\left(x_{i \min }\right)$ - the solutions that maximize (minimize) the corresponding criterion on the admissible set of solutions.

5. Write the following mappings:

(a) for the functions from set (1) to be minimized:

$$
W_{i}\left(f_{i}(X)\right)=\frac{\sum_{j=1}^{k} c_{i j} x_{j}-\sum_{j=1}^{k} c_{i j} x_{j}^{0}}{\sum_{j=1}^{k} c_{i j} x_{i \max }-\sum_{j=1}^{k} c_{i j} x_{j}^{0}} \forall i \in N_{s} ;
$$

(b) for the functions from set (1) to be maximized:

$$
W_{i}\left(f_{i}(X)\right)=\frac{\sum_{j=1}^{k} c_{i j} x_{j}^{0}-\sum_{j=1}^{k} c_{i j} x_{j}}{\sum_{j=1}^{k} c_{i j} x_{j}^{0}-\sum_{j=1}^{k} c_{i j} x_{i \min }} \forall i \in N_{m-s} .
$$


Remark. A compromise solution of this multicriterion problem is an efficient solution $x$ for which relative deviations are identical and minimum, i.e., the following condition is satisfied:

$$
\rho_{1} W_{1}(X)=\rho_{2} W_{2}(X)=\ldots=\rho_{m} W_{m}(X)=k_{0 \min } .
$$

6. Write the following one-criterion linear programming problem:

$$
k_{0}=x_{n+1} \rightarrow \min
$$

under the conditions

$$
\left\{\begin{array}{l}
\sum_{j=1}^{k} c_{i j} x_{j} \leq \sum_{j=1}^{k} c_{i j} x_{j}^{0}+\frac{k_{0}}{\rho_{i}}\left(\sum_{j=1}^{k} c_{i j} x_{i \max }-\sum_{j=1}^{k} c_{i j} x_{j}^{0}\right) \quad \forall i \in N_{s}, \\
\sum_{j=1}^{k} c_{i j} x_{j} \geq \sum_{j=1}^{k} c_{i j} x_{j}^{0}-\frac{k_{0}}{\rho_{i}}\left(\sum_{j=1}^{k} c_{i j} x_{j}^{0}-\sum_{j=1}^{k} c_{i j} x_{i \min }\right) \\
a_{i j} x_{j} \leq b_{j}, \quad i \in J_{n}, \\
\sum_{j \in \omega^{i}} x_{j} \geq \sum^{\omega^{i} \mid} g_{j}^{N_{i}} \\
\sum_{j \in \omega^{i}} x_{j} \geq \sum^{\omega^{i} \mid} g_{\eta_{i}-j+1}^{N_{i}}
\end{array}\right.
$$

7. Solve problem (18) by the dual simplex method.

8. Analyze the solution vector $x=\left(x_{1}, \ldots, x_{k}\right)$ for belonging to the set (4). If $x=\left(x_{1}, \ldots, x_{k}\right) \in E_{\eta n}^{k s}(G, H)$, then the solution is found and the algorithm is completed; otherwise, go to Step 9.

9. Check the value of $q$. If $q>1$, then go to Step 11; otherwise, go to Step 10 .

10. Increase $q$ by unity. Complement the system of constraints (18) with the cut-off inequality according to [3]

$$
\frac{x_{i_{1}}}{\Theta_{i_{1}}}+\frac{x_{i_{2}}}{\Theta_{i_{2}}}+\ldots+\frac{x_{i_{r}}}{\Theta_{i_{r}}} \geq 1
$$

as the equation

$$
-\frac{x_{i_{1}}}{\Theta_{i_{1}}}-\frac{x_{i_{2}}}{\Theta_{i_{2}}}-\ldots-\frac{x_{i_{r}}}{\Theta_{i_{r}}}+x_{n+q}=-1
$$

by introducing an auxiliary variable $x_{n+q} \geq 0$, where $i_{1}, \ldots, i_{\gamma}$ are the numbers of non-basis variables at the last point $x^{*}, \gamma$ is their quantity, and $\Theta_{i j}$ is defined $\forall j \in N_{r}$ by

$$
\Theta_{i j}=\min _{j: a_{i j}>0} \frac{b_{i}}{a_{i j}}=\frac{b_{i}}{a_{i j}} .
$$

Go to Step 6.

11. Check the value of $\Theta_{n+q-1}\left(\Theta_{n+q-1}\right.$ is defined by (21)). If $\Theta_{n+q-1} \neq 0$, go to Step 10. If $\Theta_{n+q-1}=0$, then replace the introduced auxiliary variable with zero in Eq. (20) joined to the system of equations (4), (5). Go to Step 6.

To illustrate the algorithm, let us solve the following problems.

Example 1. Let a multiset $G=\{2,2,3,3,4\}$ consisting of five elements be given. Therefore, $N_{5}=\{1,2,3,4,5\}$. Let $s=2$ be given; let us select the partition of $N_{5}$ into sets $K_{1}=\{1,3,5\}$ and $K_{2}=\{2,4\}$. Let $k=2$ be given and $k_{1}=1$ and $k_{2}=1$ be selected. Then the set is formed as $H=\{(1,2) ;(1,4) ;(3,2) ;(3,4) ;(5,2) ;(5,4)\}$. Therefore, the set of polypermutations has the form: $E_{53}^{22}(G, H)=\{(2,2) ;(2,3) ;(3,2) ;(3,3) ;(4,2) ;(4,3)\}$. 
Mathematical Formulation. Find the set of values of $x \in E_{53}^{22}(G, H)$ that are optimal for the functions

$$
\begin{gathered}
F_{1}=3 x_{1}+5 x_{2} \rightarrow \max , \\
F_{2}=-2 x_{1}-3 x_{2} \rightarrow \min .
\end{gathered}
$$

Solution. Let us use the inequalities $x_{1} \geq 2 ;-x_{1} \geq-4 ; x_{2} \geq 2 ;-x_{2} \geq-3 ; x_{1}+x_{2} \geq 4$; and $-x_{1}-x_{2} \geq-7$ to write the condition that the solution belongs to the set of polypermutations.

Objective functions will be transformed according to formulas (15) and (16). Then the minimum and maximum values of each of them can be determined. For the coefficients of the first criterion $F_{1}$, the relationship $0 \leq c_{1} \leq c_{2}$ holds; therefore, $F_{1}\left(x_{1}^{0}\right)=F_{1}(4,3)=27$ and $F_{1}\left(x_{1 \text { min }}\right)=F_{1}(2,2)=16$.

The maximum value for the function $F_{2}: F_{2}\left(x_{2}^{0}\right)=-17, F_{2}\left(x_{2} \max \right)=-10$ can be determined similarly.

As a result of transformations, we have the following functions:

$$
W_{1}=\frac{1}{2} \frac{27-3 x_{1}-5 x_{2}}{27-16}=\frac{27-3 x_{1}-5 x_{2}}{22} \leq x_{3}, \quad W_{2}=\frac{1}{2} \frac{-2 x_{1}-3 x_{2}+17}{-10+17}=\frac{-2 x_{1}-3 x_{2}+17}{14} \leq x_{3} \text {. }
$$

According to the above-stated method, we pass to the following problem:

minimize $x_{3}$ under the conditions $3 x_{1}+5 x_{2}+22 x_{3} \geq 27 ; 2 x_{1}+3 x_{2}+14 x_{3} \geq 17 ; x_{1} \geq 2 ;-x_{1} \geq-4 ; x_{2} \geq 2 ;$ $-x_{2} \geq-3 ; x_{1}+x_{2} \geq 4 ;-x_{1}-x_{2} \geq-7$.

Passage to the dual problem:

$$
27 y_{1}+17 y_{2}+2 y_{3}-4 y_{4}+2 y_{5}-3 y_{6}+4 y_{7}-7 y_{8} \rightarrow \max
$$

under the constraints

$$
\left\{\begin{array}{l}
3 y_{1}+2 y_{2}+y_{3}-y_{4}+y_{7}-y_{8} \leq 0 \\
5 y_{1}+3 y_{2}+y_{5}-y_{6}+y_{7}-y_{8} \leq 0 \\
22 y_{1}+14 y_{2} \leq 1
\end{array}\right.
$$

Solving the problem by the dual simplex method yields the following values: $x_{1}=4, x_{2}=3$ and $x_{3}=0$; $x=(4,3) \in E_{54}^{22}$ is the desired solution.

Example 2. A multiset $G=\{2,2,2,3,3,4\}$ is given, for which $N_{6}=\{1,2,3,4,5,6\} . K_{1}=\{1,2,4,6\}$ and $K_{2}=\{3,5\}$, $k_{1}=2, k_{2}=1$ are selected. Then

$$
\begin{gathered}
H=\{(1,2,3) ;(1,2,5) ;(2,1,3) ;(2,1,5) ;(1,4,3) ;(1,4,5) ;(4,1,3) ;(4,1,5) \\
\quad(1,6,3) ;(1,6,5) ;(6,1,3) ;(6,1,5) ;(2,4,3) ;(2,4,5) ;(2,4,3)(4,2,5) \\
\quad(2,6,3) ;(2,6,5) ;(2,6,3) ;(6,2,5) ;(4,6,3) ;(4,6,5) ;(6,4,3) ;(6,4,5)\} .
\end{gathered}
$$

The set of polypermutations has the form

$$
\begin{gathered}
E_{63}^{32}(G, H)=\{(2,2,2) ;(2,2,3) ;(2,2,2) ;(2,2,3) ;(2,3,2) ;(2,3,3) ;(3,2,2) \\
(3,2,3) ;(2,4,2) ;(2,4,3) ;(4,2,2) ;(2,4,3) ;(2,3,2) ;(2,3,3) ;(3,2,2) \\
(3,2,3) ;(2,4,2) ;(2,4,3) ;(4,2,2) ;(4,2,3) ;(3,4,2) ;(3,4,3) ;(4,3,3)\} \\
=\{(2,2,2) ;(2,2,3) ;(2,3,2) ;(2,3,3) ;(3,2,2) ;(3,2,3) ;(2,4,2) ;(2,4,3) \\
(4,2,2) ;(4,2,3) ;(3,4,2) ;(3,4,3) ;(4,3,3)\} .
\end{gathered}
$$

Mathematical Formulation. Find the set of values of $x \in E_{63}^{32}(G, H)$ that are optimal for the functions

$$
F_{1}=x_{1}+2 x_{2}+x_{3} \rightarrow \max , \quad F_{2}=x_{1}-3 x_{2}+3 x_{3} \rightarrow \max
$$


Solution. The system $x_{1} \geq 2 ;-x_{1} \geq-4 ; x_{2} \geq 2 ;-x_{2} \geq-4 ; x_{3} \geq 2 ;-x_{3} \geq-3 ; x_{1}+x_{2} \geq 4 ;-x_{1}-x_{2} \geq-8$; $x_{1}+x_{3} \geq 4 ;-x_{1}-x_{3} \geq-7 ; x_{2}+x_{3} \geq 4 ;-x_{2}-x_{3} \geq-7 ; x_{1}+x_{2}+x_{3} \geq 6 ;-x_{1}-x_{2}-x_{3} \geq-11$ represents the fact that the solution belongs to the set $E_{63}^{32}(G, H)$.

With Theorem 1 and formulas (15) and (16), we transform the functions as

$$
W\left(F_{1}\right)=\frac{1}{2} \cdot \frac{14-x_{1}-2 x_{2}-x_{3}}{14-8}=\frac{14-x_{1}-2 x_{2}-x_{3}}{12} \leq x_{4}, \quad W\left(F_{2}\right)=\frac{1}{2} \cdot \frac{7-x_{1}+3 x_{2}-3 x_{3}}{7+4}=\frac{7-x_{1}+3 x_{2}-3 x_{3}}{22} \leq x_{4} \text {. }
$$

We pass to the following problem: $f=x_{4} \rightarrow$ min under the conditions $x_{1}+2 x_{2}+x_{3}+12 x_{4} \geq 14 ; x_{1}-3 x_{2}+3 x_{3}$ $+22 x_{4} \geq 7 ; x_{1} \geq 2 ;-x_{1} \geq-4 ; x_{2} \geq 2 ;-x_{2} \geq-4 ; x_{3} \geq 2 ;-x_{3} \geq-3 ; x_{1}+x_{2} \geq 4 ;-x_{1}-x_{2} \geq-8 ; x_{1}+x_{3} \geq 4 ;$ $-x_{1}-x_{3} \geq-7 ; x_{2}+x_{3} \geq 4 ;-x_{2}-x_{3} \geq-7 ; x_{1}+x_{2}+x_{3} \geq 6 ;-x_{1}-x_{2}-x_{3} \geq-11$.

Let us pass to the dual problem

$$
\begin{aligned}
14 y_{1} & +7 y_{2}+2 y_{3}-4 y_{4}+2 y_{5}-4 y_{6}+2 y_{7}-3 y_{8}+4 y_{9}-8 y_{10} \\
& +4 y_{11}-7 y_{12}+4 y_{13}-7 y_{14}+6 y_{15}-11 y_{16} \rightarrow \max
\end{aligned}
$$

under the condition

$$
\left\{\begin{array}{l}
y_{1}+y_{2}+y_{3}-y_{4}+y_{9}-y_{10}+y_{11}-y_{12}+y_{15}-y_{16} \leq 0, \\
2 y_{1}-3 y_{2}+y_{5}-y_{6}+y_{9}-y_{10}+y_{13}-y_{14}+y_{15}-y_{16} \leq 0 \\
y_{1}+3 y_{2}+y_{7}-y_{8}+y_{11}-y_{12}+y_{13}-y_{14}+y_{15}-y_{16} \leq 0, \\
12 y_{1}+22 y_{2} \leq 1 .
\end{array}\right.
$$

Solving the problem by the simplex method yields $x_{1}=4 ; x_{2}=2,8 ; x_{3}=3 ;$ and $x_{4}=0,1$.

The cut-off is carried out and the point $x^{*}=(4,3,3)$ is obtained, which is optimal for the functions.

Remark. This algorithm converges to the optimal solution in a finite number of steps. Since the set of admissible solutions $X$ is bounded, the problem (2), (4), (5) has a finite optimal solution.

Thus, we have developed and substantiated a possible approach to the solution of multicriterion problems. A solution algorithm has been developed and implemented. Numerical experiments have been carried out. The subsequent studies are planned to study the efficiency of the existing method of solving multicriterion problems and to develop new solution techniques.

\section{REFERENCES}

1. I. V. Sergienko and V. P. Shilo, Discrete Optimization Problems: Challenges, Solution Techniques, Analysis [in Russian], Naukova Dumka, Kyiv (2003).

2. Yu. P. Zaichenko, Operations Research. Fuzzy Optimization: A Textbook [in Russian], Vyshcha Shkola, Kyiv (1991).

3. O. O. Yemets and L. M. Kolechkina, Combinatorial Optimization Problems with Fractional-Linear Objective Functions [in Ukrainian], Naukova Dumka, Kyiv (2005).

4. Yu. G. Stoyan and O. O. Yemets, Theory and Methods of Euclidean Combinatorial Optimization [in Ukrainian], Kyiv (1993).

5. V. V. Podinovskii and V. D. Nogin, Pareto-Optimal Solutions to Multicriterion Problems [in Russian], Nauka, Moscow (1982).

6. I. V. Sergienko, L. N. Kozeratskaya, and T. T. Lebedeva, Stability and Parametric Analyses of Discrete Optimization Problems [in Russian], Naukova Dumka, Kyiv (1995).

7. T. T. Lebedeva, N. V. Semenova, and T. I. Sergienko, "Stability of vector problems of integer optimization: Relationship with the stability of sets of optimal and nonoptimal solutions," Cybern. Syst. Analysis, 41, No. 4, 551-558 (2005).

8. Yu. G. Stoyan, O. O. Yemets, and E. M. Yemets, Optimization on Polypermutations: Theory and Methods [in Ukrainian], RVTs PUSKU, Poltava (2005). 